\section{AB0194 ASSOCIATION OF MALNUTRITION WITH QUALITY OF LIFE IN ELDERLY RA PATIENTS}

W. Tański ${ }^{1}$, J. Wójciga ${ }^{2}$, N. Świątoniowska-Lonc ${ }^{3}$, B. Jankowska-Polańska ${ }^{3,4}$. ${ }^{1} 4$ th Military Teaching Hospital, Department of Internal Medicine, Wrocław, Poland; ${ }^{2}$ Wroclaw Medical University, Student Research Club at the Division of Nursing in Internal, Wroclaw, Poland; ${ }^{3}$ Wroclaw Medical University, Department of Clinical Nursing, Wroclaw, Poland; ${ }^{4} 4$ th Military Teaching Hospital, Research and Development Department, Wroclaw, Poland

Background: Rheumatoid arthritis (RA) is a progressive joint condition that leads to joints destruction and complications in the internal organs and significantly affects the a patient's functional ability. Elderly patients with RA complain of pain, mood and sleep disturbances, fatigue, and insomnia in addition to weakness, decreased appetite and weight loss. Chronic use of medications results in the risk of comorbidities and decreased physical functioning. All this together significantly contributes to a negative assessment of quality of life (QoL).

Objectives: Due to the chronic nature of RA and the high risk of malnutrition in this group of patients, an attempt was made to assess the QoL, activities of daily living and severity of frailty syndrome according to the results of the MNA questionnaire in a group of elderly patients with RA.

Methods: The study included 98 patients (age over 60 years) with a diagnosis of RA according to ARA. Standardized tools were used in the study: the WHOQOL-BREF to assess quality of life, the Edmonton Frailty Scale to assess frailty syndrome, MNA to assess nutritional status and MMSE to assess cognitive function. Medical data were taken from the hospital records.

Results: Patients significantly differed in the extent of limitations in basic (ADL) and advanced activities of daily living (IADL) according to their nutritional status. The higher the level of malnutrition, the greater the limitations of undertaken activities. In addition, an adverse effect of reduced body weight on the occurrence of cognitive dysfunction was observed $(33.33 \%$ of malnourished patients were diagnosed with dementia vs. $1.79 \%$ in normal weight patients). Similarly, frailty syndrome or vulnerability to frailty syndrome was associated with malnourished patients $(33.3 \%$ mild, $16.67 \%$ moderate, and $16.67 \%$ severe frailty syndrome). Malnourished patients had significantly lower QoL scores in all domains of the WHOQOL-BREF questionnaire compared to normal weight patients and multivariate analysis of the effect of selected variables on QoL in the domains of the WHOQOL-BREF questionnaire showed that a significant independent determinant of lower QoL in all domains was the presence of frailty syndrome, respectively: Perception of $\mathrm{QoL}(\mathrm{R}=-0$. 069), Self-perception of health $(R=-0.172)$, Physical domain $(R=-0.425)$, Psychological domain $(R=-0.432)$, Social domain $(R=-0.415)$, Environmental domain $(\mathrm{R}=-0.317)$. Malnutrition was a significant independent determinant of $Q \mathrm{oL}$ in the self-perception of health domain $(R=-0.08)$. Additionally, in the regression analysis, a positive effect of male gender was observed on the assessment of $Q \circ L$ in the psychological $(R=1.414)$ and environmental $(R=1.123)$ domains.

Table 5. Comparative analysis of quality of life in terms of each domain of the WHOQOL-BREF questionnaire according to nutritional status.

\begin{tabular}{lcccc}
\hline & \multicolumn{4}{c}{ MNA } \\
\cline { 2 - 4 } & $\begin{array}{c}\text { Malnutrition } \\
\text { (Mean } \pm \text { SD) }\end{array}$ & $\begin{array}{c}\text { Risk of malnutri- } \\
\text { tion (Mean } \pm \text { SD) }\end{array}$ & $\begin{array}{c}\text { Normal nutrition } \\
\text { (Mean } \pm \text { SD) }\end{array}$ & $\mathrm{p}$ \\
\hline WHOQOL BREF & $3,33 \pm 1,03$ & $3,33 \pm 0,68$ & $3,68 \pm 0,58$ & 0,029 \\
Perception of quality of life & $2,67 \pm 0,82$ & $2,47 \pm 0,84$ & $3,14 \pm 0,98$ & 0,004 \\
Self-perception of health & $10,83 \pm 3,49$ & $11,31 \pm 2,39$ & $12,68 \pm 2,27$ & 0,02 \\
Physical health & $12,5 \pm 3,89$ & $13,28 \pm 2,73$ & $14,45 \pm 2,24$ & 0,056 \\
Psychological domain & $12,33 \pm 2,86$ & $12,67 \pm 2,93$ & $14,29 \pm 2,61$ & 0,007 \\
Social domain & $13,83 \pm 2,86$ & $13,22 \pm 2,28$ & $13,96 \pm 2,17$ & 0,34 \\
Environmental domain & & & & \\
\hline
\end{tabular}

Conclusion: Malnourished people have a lower quality of life than people of normal weight. Malnutrition is a factor that negatively affects daily functioning, cognitive functioning, and the severity of frailty syndrome. A significant independent determinant of reduced quality of life across all domains of the WHOQOL-BREF questionnaire is frailty syndrome.

REFERENCES:

[1] Marcora SM, Chester KR, Mittal G et al. Randomizedphase 2 trial of anti-tumor necrosisfactortherapy for cachexia in patients with earlyrheumatoidarthritis. Am J ClinNutr 2006;84:1463-72. 24 .

[2] Kremers HM, Nicola PJ, Crowson CS, et al. Prognosticimportance of low body mass index in relation to cardiovascularmortality in rheumatoidarthritis. ArthritisRheum. 2004;50:3450-7.

Disclosure of Interests: None declared

DOI: 10.1136/annrheumdis-2021-eular.4297

\section{Rheumatoid arthritis - biological DMARDs}

\begin{tabular}{|l|l|}
\hline AB0195 & PHARMACOKINETIC AND PHARMACODYNAMIC \\
& EVALUATION OF A PROPOSED BIOSIMILAR \\
& MSB11456 VERSUS BOTH THE US-LICENSED AND \\
& EU-APPROVED TOCILIZUMAB: RESULTS OF A \\
& RANDOMIZED, DOUBLE-BLIND, PARALLEL-GROUP, \\
& SINGLE-DOSE TRIAL IN HEALTHY ADULTS
\end{tabular}

C. Schwabe ${ }^{1}$, C. Wynne ${ }^{2}$, A. Illes ${ }^{3}$, M. Ullmann ${ }^{3}$, E. Vincent ${ }^{3}$, V. Ghori ${ }^{3}$, C. PetitFrere ${ }^{3}$, A. S. Racault ${ }^{3}$, I. Morin ${ }^{3} .{ }^{1}$ Auckland Clinical Studies Ltd, Auckland, New Zealand; ${ }^{2}$ Christchurch Clinical Studies Trust Ltd, Christchurch, New Zealand; ${ }^{3}$ Biosimilars, Fresenius Kabi SwissBioSim, Eysins, Switzerland

Background: Tocilizumab, a biologic disease-modifying antirheumatic drug, is a recombinant humanized monoclonal immunoglobulin $\mathrm{G} 1$ antibody against the interleukin-6 receptor (IL-6R). MSB11456 is a proposed biosimilar to the US-licensed tocilizumab and EU-approved tocilizumab. Prior to initiation of its clinical development program, MSB11456 was considered highly similar to the reference products based on extensive in vitro pharmacological testing and functiona activity assays.

Objectives: This double-blind, parallel-group phase I study (NCT03282851) assessed the pharmacokinetic (PK) and pharmacodynamic (PD) equivalence of MSB11456 to US-licensed and EU-approved tocilizumab and provided data on the similarity of safety profiles and immunogenicity of MSB11456 and the reference products in healthy adult subjects.

Methods: Healthy adult volunteers were randomized to receive a single $162 \mathrm{mg}$ subcutaneous injection of MSB11456, US-licensed tocilizumab, or EU-approved tocilizumab. Samples for PK/PD and immunogenicity analysis were taken predose, up to 29 days postdose, and at the end of study visit (day 48). Primary endpoint $\mathrm{PK}$ parameters were natural log transformed and analyzed using analysis of covariance (ANCOVA) methods; results were then transformed back to the original scale. Secondary PD analysis measured serum soluble IL-6R and serum $C$ reactive protein (sCRP) parameters, and data were analyzed using a method similar to that for PK data. Safety data were collected throughout the study and analyzed descriptively.

Results: 685 subjects were randomized and treated. Results of the primary PK analysis demonstrated bioequivalence between MSB11456 and both US-licensed and EU-approved tocilizumab, as well as between the reference products, since all corresponding $90 \%$ confidence intervals (Cls) for the geometric least squares mean (LSM) ratios were within the predefined $80.00 \%$ to $125.00 \%$ equivalence margin (Table 1). PD analyses also demonstrated equivalence of MSB11456 and both US-licensed and EU-approved tocilizumab, as well as between the reference products. Safety, tolerability and immunogenicity were also comparable between treatments. The incidence of tocilizumab-specific neutralizing antibodies was low (<3\% for all three products).

Conclusion: PK and PD equivalence of MSB11456, US-licensed tocilizumab, and EU-approved tocilizumab were demonstrated with comparable immunogenicity, safety, and tolerability for the three products. This study adds to the totality of evidence in support of MSB11456 as a proposed biosimilar to tocilizumab.

Table 1. Results of primary PK analysis

\begin{tabular}{|c|c|c|c|c|}
\hline Parameter & Treatment & $\mathrm{n}$ & Ratio of geometric LSMs (\%) & $90 \% \mathrm{Cl}$ of ratio \\
\hline \multicolumn{5}{|c|}{ MSB11456 vs US-licensed tocilizumab } \\
\hline \multirow{2}{*}{$\mathrm{AUC}_{0-\infty}$} & MSB11456 & 194 & 106.16 & $96.80,116.43$ \\
\hline & US-licensed tocilizumab & 190 & & \\
\hline \multirow{2}{*}{$\mathrm{AUC}_{0-\mathrm{t}}$} & MSB11456 & 230 & 104.15 & $93.58,115.90$ \\
\hline & US-licensed tocilizumab & 226 & & \\
\hline \multirow[t]{2}{*}{$\mathrm{C}_{\max }$} & MSB11456 & 230 & 104.45 & $95.05,114.77$ \\
\hline & US-licensed tocilizumab & 226 & & \\
\hline \multicolumn{5}{|c|}{ MSB11456 vs EU-tocilizumab } \\
\hline \multirow{2}{*}{$\mathrm{AUC}_{0-\infty}$} & MSB11456 & 194 & 104.03 & $94.96,113.96$ \\
\hline & EU-approved tocilizumab & 200 & & \\
\hline \multirow{2}{*}{$A \cup C_{0-t}$} & MSB11456 & 230 & 94.78 & $85.15,105.50$ \\
\hline & EU-approved tocilizumab & 224 & & \\
\hline \multirow{2}{*}{$\mathrm{C}_{\max }$} & MSB11456 & 230 & 94.83 & $86.28,104.22$ \\
\hline & EU-approved tocilizumab & 224 & & \\
\hline \multicolumn{5}{|c|}{ US-licensed vs EU-approved tocilizumab } \\
\hline \multirow[t]{2}{*}{$\mathrm{AUC}_{0-\infty}$} & US-licensed tocilizumab & 190 & 97.99 & $89.40,107.41$ \\
\hline & EU-approved tocilizumab & 200 & & \\
\hline \multirow{2}{*}{$\mathrm{AUC}_{0-\mathrm{t}}$} & US-licensed tocilizumab & 226 & 91.01 & $81.71,101.36$ \\
\hline & EU-approved tocilizumab & 224 & & \\
\hline \multirow{2}{*}{$\mathrm{C}_{\max }$} & US-licensed tocilizumab & 226 & 90.79 & $82.57,99.84$ \\
\hline & EU-approved tocilizumab & 224 & & \\
\hline
\end{tabular}

AUC, area under the serum concentration-time curve $(\mu \mathrm{g} \cdot \mathrm{h} / \mathrm{mL}) ; \mathrm{AUC}, \mathrm{AUC}$ from time zero to infinity; $A \cup C_{0-t}, A U C$ from time zero to the time of the last quantifiable concentration; $C_{\max }$ maximum concentration $(\mu \mathrm{g} / \mathrm{mL})$ 